\title{
Dense poly(ethylene glycol) coatings maximize nanoparticle transport across lymphatic endothelial cells
}

Jacob McCright ${ }^{1}$, Colin Skeen ${ }^{1}$, Jenny Yarmovsky ${ }^{1}$, Katharina Maisel ${ }^{1 *}$

${ }^{1}$ Department of Bioengineering, University of Maryland College Park, College Park, MD, USA *corresponding author

Send correspondence to:

Dr. Katharina Maisel: maiselka@umd.edu

8278 Paint Branch Drive

University of Maryland

College Park, MD 20742 


\begin{abstract}
:
Lymphatic vessels have received considerable attention in recent years as delivery route for immune modulatory therapies to the lymph nodes. Lymph node targeting of immunotherapies and vaccines has been shown to significantly enhance their therapeutic efficacy. Lymphatics transport functions materials from peripheral tissues to the lymph nodes, including small $10-250 \mathrm{~nm}$ therapeutic nanoparticles. While size required to enter lymphatic vessels, surface chemistry is more poorly studied. Here, we probed the effects of surface poly(ethylene glycol) (PEG) density on nanoparticle transport across lymphatic endothelial cells (LECs). We differentially PEGylated model carboxylate-modified polystyrene nanoparticles to form either a brush or dense brush PEG conformation on the nanoparticle surfaces. Using an established in-vitro lymphatic transport model, we found that the addition of any PEG improved the transport of nanoparticles through lymphatic endothelial cells (2.5 - $2.6 \pm 0.9 \%$ transport efficiency at 24 hours) compared to the unmodified PS-COOH nanoparticles (0.05 $\pm 0.05 \%$ transport efficiency at 24 hours). Additionally, we found that transcellular transport is maximized (4.2 $\pm 0.7 \%$ transport efficiency at 24 hours) when the PEG is in a dense brush conformation on nanoparticle surfaces, corresponding with a high grafting density $\left(R_{f} / D=4.9\right)$. These results suggest that $P E G$ conformation has a crucial role in determining translocation of nanoparticles across LECs and into lymphatic vessels. Thus, we identified PEG density as a major design criteria for maximizing lymphatic targeting of therapeutic nanoparticle formulations that can be widely applied to enhance immunotherapeutic and vaccine outcomes in future studies.
\end{abstract}




\section{Introduction}

Lymphatic vessels exist throughout the entire body and are known for transporting cells, fluid, and particulates from peripheral tissues to the local draining lymph nodes (LNs), where the adaptive immune response is formed [1]. In recent years lymphatics have received increasing attention as potential drug delivery targets to transport immune modulatory therapies to the LNs without requiring direct injections. Delivering immunotherapies, including vaccines, to the LNs has been shown to potentiate their therapeutic effects, particularly crucial as efficacy of many immunotherapies still require improvement. Recent studies have demonstrated that nanoparticles between $10-250 \mathrm{~nm}$ are transported preferentially via lymphatic vessels from peripheral tissues to LNs, highlighting that the transport functions of lymphatics can be taken advantage for drug delivery [2-5].

While size required for lymphatic entry is well established, conflicting data about the nanoparticle surface chemistry required to maximize lymphatic transport exist. A study comparing cationic liposomes and anionic poly(lactic-co-glycolic) (PLGA) nanoparticles demonstrated that cationic liposomes accumulate to a greater extent in the LN after subcutaneous injection compared to anionic PLGA nanoparticles [5]. However, there is large size discrepancy between the two systems: cationic $180 \mathrm{~nm}$ liposomes were well within the lymphatic targeting size range while anionic $350 \mathrm{~nm}$ PLGA nanoparticles were likely too large to preferentially enter lymphatic vessels. Thus, the question about surface chemistry effects remains unanswered. Another study demonstrated that positively charged $30 \mathrm{~nm}$ polyethyleneimine-stearic acid micelles preferentially accumulated in draining LNs compared to free antigen [6]. Another group demonstrated that coating $200 \mathrm{~nm}$ poly(methacrylate) (PMA) nanoparticles with PEG markedly improved LN accumulation of nanoparticles after 12 and 48 hours [7]. Yet another study found that 50, 100, and $200 \mathrm{~nm}$ polyethylene glycol (PEG) coated nanoparticles accumulated more in the LNs after subcutaneous injection compared to uncoated PLGA nanoparticles of the same size, suggesting that hydrophilicity is crucial to maximize lymphatic transport of nanoparticles [8]. However, in this 
study, the surface potential of PEGylated nanoparticles was only $-36.1 \pm 14.6 \mathrm{mV}$, suggesting that the PEG coating was very not dense. Combined, these results suggest that hydrophilicity through addition of e.g. PEG may be beneficial for enhancing nanoparticle transport by lymphatics, but also highlight the importance to more critically assess the effect of surface chemistry on their transport by lymphatic vessels.

PEGylating nanoparticles is a strategy that has been used extensively to enhance nanoparticle interactions with biological materials. Most notably, chemotherapeutic drug doxorubicin formulated as PEGylated liposomes is one of few FDA-approved nanoparticle treatments. The addition of PEG enhanced the circulation time of the chemotherapeutic was enhanced, improving the overall efficacy [9-14]. PEG coatings can be optimized by modulating the density and molecular weight (MW) of PEG on the surface of nanoparticles. As PEG density on the surface of a nanoparticle is increased, the conformation the PEG takes transitions from a "mushroom" conformation to a "dense brush" as steric hindrances restrict movement and self-coiling of the grafted polymer [15]. PEG conformation has been shown to be critical in formulating nanoparticles that effectively cross biological barriers. For example, to cross the mucus barrier, researchers have found that nanoparticles need to be in the dense brush conformation, which can be achieved by densely coating nanoparticles with short $2-20$ kDa PEG chains [16]. Similarly, penetration through brain tissues has been achieved by coating small $<100 \mathrm{~nm}$ nanoparticles with PEG in the dense brush conformation [17]. Tumor penetration has also been improved by PEGylating nanoparticles: the addition of PEG to the surface of model $60 \mathrm{~nm}$ negatively charged polystyrene (PS) nanoparticles enhanced nanoparticle diffusion through breast cancer xenograft slices ex vivo [18]. This study also showed that increasing the surface density of the PEG coating from $3.7 \pm$ 1.6 PEG/100nm² (mushroom conformation) to $15.7 \pm 8.6 \mathrm{PEG} / 100 \mathrm{~nm}^{2}$ (intermediate brush) further improved diffusion through tumor tissue. PEGylation of nanoparticles has also been shown to enhance nanoparticle-mediated biofilm disruption. Recently, PEGylated $11 \mathrm{~nm}$ gold and $8 \mathrm{~nm}$ iron oxide nanoparticles were able to reduce the biomass of biofilms produced by Legionella 
pneumophila compared to citrate-coated $8 \mathrm{~nm}$ silver nanoparticles. The enhanced anti-biofilm properties of the nanoparticles was attributed to the improved colloidal stability and diffusion through the biofilm conferred by the addition of PEG to the nanoparticle surfaces [19]. PEGylation has also been crucial to provide stealth characteristics in systemic circulation. Studies have shown that circulation time of $100 \mathrm{~nm}$ anionic, hydrophobic nanoparticles that displayed rapid clearance from the bloodstream, could be markedly improved through the addition of $5 \mathrm{kDa}$ PEG in density dependent manner [20]. Additionally, PEG densities greater than $1.5 \mathrm{PEG} / \mathrm{nm}^{2}$, indicating a "brush" conformation, remained in circulation longer than nanoparticles with surface PEG densities in "mushroom" conformation [20]. Combined, these results demonstrate that the conformation of PEG on the surface is a key parameter in nanoparticle formulations. However, what PEG density maximizes nanoparticle transport by lymphatic vessels remains to be explored. Here, we investigated the effect of PEG surface density on nanoparticle transport by lymphatic vessels and identify the PEG density required to maximize this transport. We generated a library of nanoparticles coated with varying PEG densities, and thus varying PEG conformations, and tested their transport by lymphatic endothelial cells using a previously established in vitro lymphatic transport model that recapitulates in vivo transport [21]. The nanoparticle design criteria resulting from our studies have the potential to significantly enhance efficacy of immunotherapies by maximizing nanoparticle transport to the LNs via lymphatic vessels.

\section{Methods}

\section{Nanoparticle formulation}

$100 \mathrm{~nm}$ red fluorescent carboxyl $(\mathrm{COOH})$ modified polystyrene (PS) nanoparticles (Thermo Fisher Scientific, F8801) were covalently modified with 5 kDa MW methoxy (MeO)-PEG-amine $\left(\mathrm{NH}_{2}\right)$ (Creative PEGworks) using a previously described method [17]. Briefly, PS particle were suspended at $0.1 \% \mathrm{w} / \mathrm{v}$ in $200 \mathrm{mM}$ borate buffer $(\mathrm{pH}=8.2)$. Nanoparticles were generated with the following concentrations of PEG: $350 \mu \mathrm{M}$ (theoretical 100\% PEG coverage of COOH groups, PSPEG-100), $175 \mu \mathrm{M}$ (50\% COOH groups, PSPEG-50), $87.5 \mu \mathrm{M}$ (25\% COOH groups, PSPEG- 
25), and $35 \mu \mathrm{M}(10 \% \mathrm{COOH}$ groups, PSPEG-10). PEG was conjugated to nanoparticles using 7 mM N-Hydroxysulfosuccinimide (NHS) (Sigma) and 0.02 mM 1-Ethyl-3-(3-dimethylaminopropyl) carbodiimide (EDC) (Invitrogen). Reaction was allowed to proceed on a rotary incubator at room temperature for at least 4 hours. Nanoparticles were collected using 100k MWCO centrifugal filters (Amicon Ultra; Millipore) and washed with de-ionized (DI) water. Nanoparticles were resuspended at $1 \% \mathrm{w} / \mathrm{v}$ in $\mathrm{DI}$ water stored at $4^{\circ} \mathrm{C}$.

Nanoparticle characterization

Dynamic light scattering (DLS) was used to measure the hydrodynamic diameter and polydispersity index (PDI) of nanoparticles. Phase analysis light scattering (PALS) was used for measuring $\zeta$ - potential (NanoBrook Omni). Measurements were performed using a scattering angle of $90^{\circ}$ at $25^{\circ} \mathrm{C}$.

\section{PEG density characterization}

PEG density was determined using a previously published method [22]. Briefly, 5kDa PEG-NH2 (Creative PEGworks) conjugated to fluorescein isothiocyanate (FITC) was conjugated to red fluorescent $100 \mathrm{~nm}$ carboxyl modified nanoparticles. A FITC-PEG-NH $\mathrm{N}_{2}$ standard curve was generated in DI water to calculate the PEG amount on the nanoparticle surface using a plate reader (Tecan Spark Multimode Microplate Reader). From these measurements, PEG grafting distance (D) can be calculated and PEG density estimated using the Flory radius of PEG $\left(R_{\mathrm{f}}\right)$. The Flory radius of a polymer chains is defined as $R_{f} \sim \mathrm{aN}^{3 / 5}$, where $N$ is the degree of polymerization, and $\alpha$ is the effective monomer length. An unconstrained $5 \mathrm{kDa}$ PEG chain has a $\mathrm{R}_{\mathrm{f}}$ of $5.4 \mathrm{~nm}$ and occupies $22.7 \mathrm{~nm}^{2}$. PEG density and conformation can be correlated to the ratio of $R_{f} / D$, with $R_{f} / D<1$ yielding a mushroom conformation, $1<R_{f} / D>4$ yielding a brush conformation, and $R_{f} / D>4$ yielding a dense brush.

\section{Nanoparticle uptake}

Immortalized human lymphatic endothelial cells (hiLECs, [23]) were seeded at a density of 200,000 cells $/ \mathrm{cm}^{2}$ onto collagen (Corning) coated plates and cultured in endothelial growth media 
-2 (EGM2, Lonza) at $37^{\circ} \mathrm{C}$ and $5 \% \mathrm{CO}_{2}$ overnight. hiLECs were incubated with nanoparticle at $0.05 \% \mathrm{w} / \mathrm{v}$ for $3 \mathrm{~h}$ and uptake was assessed either by flow cytometry or fluorescence microscopy. For fluorescence microscopy, samples were fixed with $2 \%$ paraformaldehyde(ThermoFisher) and imaged using a Zeiss Axio Observer. For flow cytometry, cells were released from the substrate using Accutase® (Innovative Cell Technologies), fixed with 2\% paraformaldehyde, and flow cytometry was performed using a BD FACSelecta. Data was analyzed using FlowJo software (Tree Star) and FIJI (ImageJ).

\section{Lymphatic transport model}

Nanoparticle transport across lymphatic endothelial cells (LEC) was assessed using an established in vitro model that recapitulates in vivo lymphatic transport [21]. Briefly, human primary LECs (hLECs) were seeded on $1.0 \mu \mathrm{m}$ pore size $12 \mathrm{~mm}$ transwell inserts (Falcon) at 200,000 cells $/ \mathrm{cm}^{2}$ and cultured in EGM-2 (Lonza) at $37^{\circ} \mathrm{C}$ and $5 \% \mathrm{CO}_{2}$ for $48 \mathrm{~h}$. Cells were pretreated with $1 \mu \mathrm{m} / \mathrm{s}$ transmural flow to simulate the tissue microenvironment. hLECs were treated with $1 \% \mathrm{w} / \mathrm{v}$ nanoparticles on the apical side and the basolateral compartment was sampled every $3 \mathrm{~h}$ for up to $24 \mathrm{~h}$. Fluorescence intensity was measured using a plate reader (Tecan) and the amount of nanoparticles transported was calculated using a standard curve. Transport experiments were performed in EGM2 without growth factors to avoid confounding effects of growth factors on transport mechanisms. Effective permeability was estimated using the following equation: $\boldsymbol{P}_{\text {eff }}=\frac{\boldsymbol{C}_{\text {lower }} V_{\text {lower }}}{\boldsymbol{t S C}_{\text {initial }}}$, where $\mathrm{C}=$ concentration, $\mathrm{V}_{\text {lower }}=$ volume of the basolateral compartment, $\mathrm{S}=$ surface area, and $\mathrm{t}=$ time. $\mathrm{hLEC}$ monolayer formation was confirmed after experiments using immunofluorescence.

\section{Immunofluorescence staining}

Cells were fixed in $2 \%$ PFA for 15 minutes and incubated with mouse anti-human VE-Cadherin (BD Sciences) at $4^{\circ} \mathrm{C}$ overnight. Secondary antibodies conjugated to Alexa Fluor® 488 or 647 were used for detection (Thermo Fisher). Slides were mounted using DAPI (4',6-diamidino-2- 
phenylindole)-containing Vectashield (Vector Laboratories Inc., Burlingame, CA) and imaged using a Zeiss Axio Observer. Image processing was performed using FIJI (NIH).

\section{Statistics}

Group analysis was performed using a 2-way ANOVA, followed by Tukey's post-test. Unpaired Student's t-test was used to examine differences between only two groups. A value of $p<0.05$ was considered significant (GraphPad). All data is presented as mean \pm standard error of the mean.

\section{Results}

Increasing PEG density on nanoparticles neutralizes surface $\zeta$-potential

The conformation of PEG on the surface of nanoparticles has been shown to affect how the nanoparticle interacts with surrounding tissues and cells. In this study we generated differentially PEGylated nanoparticles to determine how tuning PEG grafting density modulated surface PEG conformation as well as nanoparticle surface charge. As expected, we found that increasing PEG density on the surface PSPEG nanoparticles increased their diameter and neutralized their previously negative surface charge. Unmodified PS nanoparticles had a diameter of $108 \pm 1 \mathrm{~nm}$ and a $\zeta$-potential of $-22.4 \pm 3.3 \mathrm{mV}$ (Fig 1A). Addition of PEG increased the nanoparticle diameter to >120 nm (Fig 1A). Increasing PEGylation resulted in decreased nanoparticle $\zeta$-potentials: the densest PEGylated nanoparticles, PSPEG-100 had a $\zeta$-potential of $-2.9 \pm 2.5 \mathrm{mV}$, while less densely PEGylated PSPEG-50 had $\zeta$-potential of $-5.1 \pm 3.5 \mathrm{mV}$, PSPEG-25 had a $\zeta$-potential of $4.7 \pm 2.5 \mathrm{mV}$, and PSPEG-10 had a $\zeta$-potential of $-10.2 \pm 6.6 \mathrm{mV}$. Un-PEGylated PS-COOH nanoparticles had $\zeta$-potential of $-41.9 \pm 5.9 \mathrm{mV}$.

To assess PEG density, we then quantified the ratio of Flory radius $\left(R_{f}\right)$ of the polymer to the measured grafting distance (D). Different PEGylation yielded varying PEG density on nanoparticles, with PSPEG-100 having Rf/D of $4.9 \pm 0.1$, PSPEG-50 having Rf/D $2.4 \pm 0.1$, PSPEG-50 having $R_{f} / D$ of $1.7 \pm 0.1$, and PSPEG-10 having $R_{f} / D$ of $1.3 \pm 0.1$ (Fig 1B). These $\mathrm{Rf} / \mathrm{D}$ values can be correlated to the conformation of PEG on the nanoparticle surface, with $\mathrm{Rf} / \mathrm{D}$ 
A

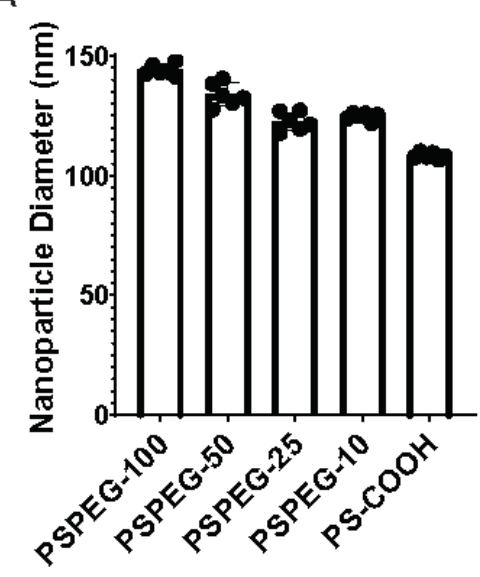

B

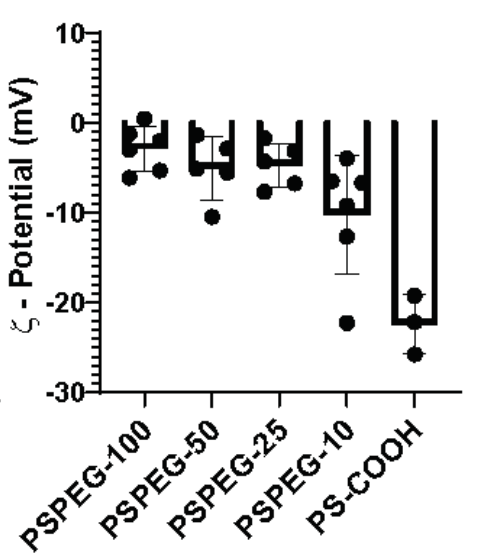

C
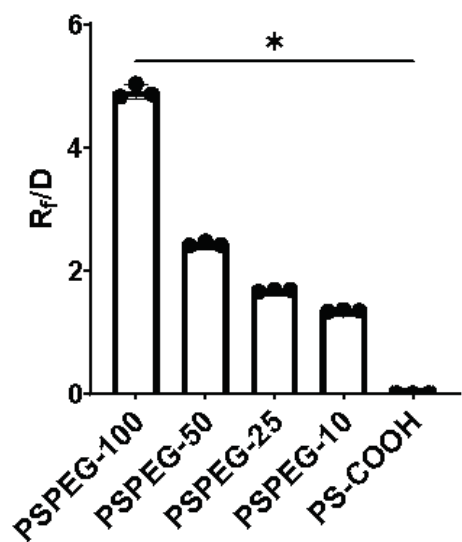

D

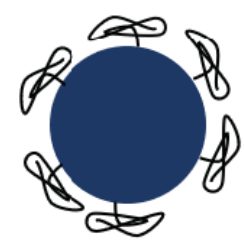

Mushroom conformation $\mathrm{R}_{\mathrm{f}} / \mathrm{D}<1$

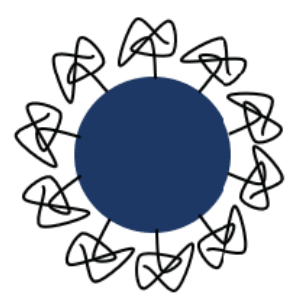

Intermediate brush conformation $1<\mathrm{R}_{\mathrm{f}} / \mathrm{D}<4$

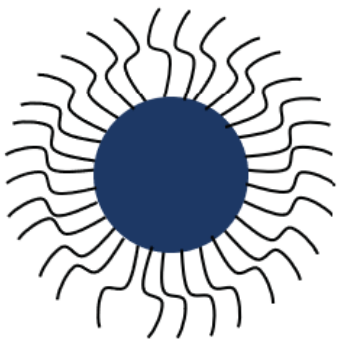

Dense brush conformation $\mathrm{R}_{\mathrm{f}} / \mathrm{D}>4$

Figure 1: PEG grafted onto nanoparticles at different densities reduces surface charge.

A) Dynamic Light Scattering (DLS) measurement of nanoparticle hydrodynamic diameters and B) Phase Analysis Light Scattering (PALS) measurement of nanoparticle $\zeta$ - potentials. C) $R_{f} / D$ values of nanoparticles indicating nanoparticle PEG density and conformation. D) Schematic of $P E G$ conformation based on $R_{f} / D$ on a spherical nanoparticle.

$<1$ indicating a mushroom conformation, $1<\mathrm{Rf} / \mathrm{D}<4$ indicating an intermediate brush conformation, and Rf/D > 4 indicating a dense brush conformation (Fig 1C-D). Here, we generated nanoparticles with intermediate brush (PSPEG-50, PSPEG-25, PSPEG-10) and dense brush (PSPEG-100) conformation.

Surface PEG density does not affect nanoparticle uptake by lymphatics

We next sought to investigate how PEG density on nanoparticles affects their uptake by LECs, as this is the first step in transcellular transport of materials. Using fluorescence microscopy (Fig 2A), we found that hiLECs containing fluorescently labeled nanoparticles had a normalized median fluorescence intensities (MFI) of the $1.16 \pm 0.36$ for PSPEG-100 (dense brush), $0.93 \pm$ 0.27 for PSPEG-50, $0.91 \pm 0.23$ for PSPEG-25, and $0.93 \pm 0.30$ for PSPEG-10. HiLECs 
containing unmodified

\section{PS- $\mathrm{COOH}$}

nanoparticles had an

MFI of $1.15 \pm 0.23$ (Fig

2A). Flow cytometry

showed that $>85 \%$ of

hiLECs take up PS and

PSPEG nanoparticles,

confirming imaging data.

These results suggest

that nanoparticle surface
A Nanoparticle Uptake by hiLECs

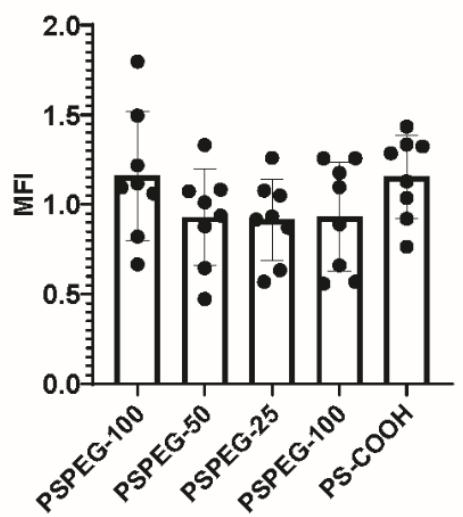

B Nanoparticle Uptake by hiLECs

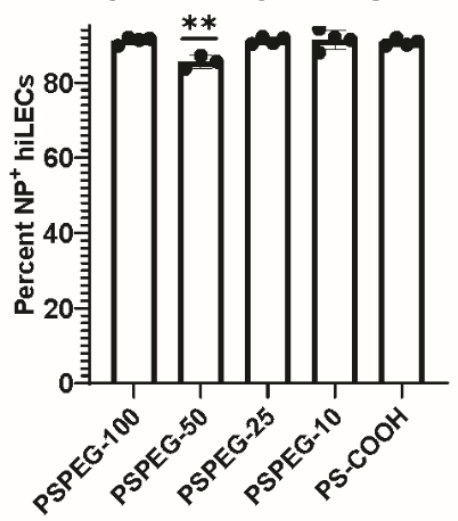

Figure 2: PEG density does not affect nanoparticle uptake by hiLECs. A) Mean fluorescent intensity (MFI) extracted from fluorescent images of hiLECs cultured with PSPEG nanoparticles. B) Percent of hiLECs containing PSPEG nanoparticles after threehour incubation as measured using flow cytometry.

PEG conformation does not significantly affect nanoparticle uptake by hiLECs.

Dense brush PEG coatings on nanoparticles maximize their transport across LECs

We next sought to assess A

the effect of PEG density on nanoparticle transport by lymphatics, using an in vitro transendothelial transport
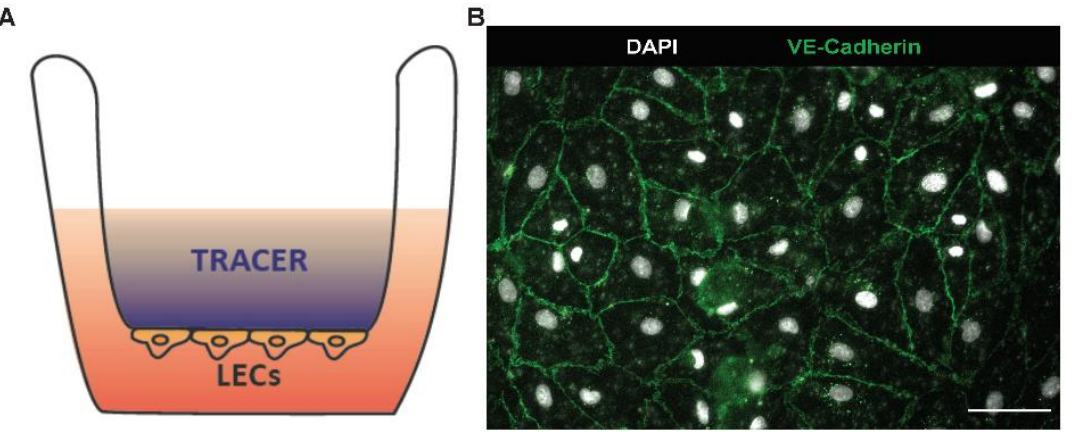

model (Fig 3A). We confirmed formation of a robust monolayer using intercellular junction marker

Figure 3: Lymphatic transport model. A) Schematic showing primary hLECs cultured on a transwell insert with fluorescent tracer, such as nanoparticles, on the apical side. B) hLECs form a monolayer on transwells, confirmed through VE-cadherin staining (green). Scale bar $40 \mu \mathrm{m}$.

VE-Cadherin, a primary indicator of endothelial cell permeability (Fig 3B). We found that the unmodified PS-COOH nanoparticles were minimally transported across hLECs, while PSPEG100 were transported significantly more by $6 \mathrm{~h}$ (Fig 4A), and by $24 \mathrm{~h}$ there was a $\sim 90$ fold increase in transport, with $4.2 \pm 0.7 \%$ transported for PSPEG-100 vs $0.05 \pm 0.05 \%$ for PS-COOH (Fig 4A- 
A

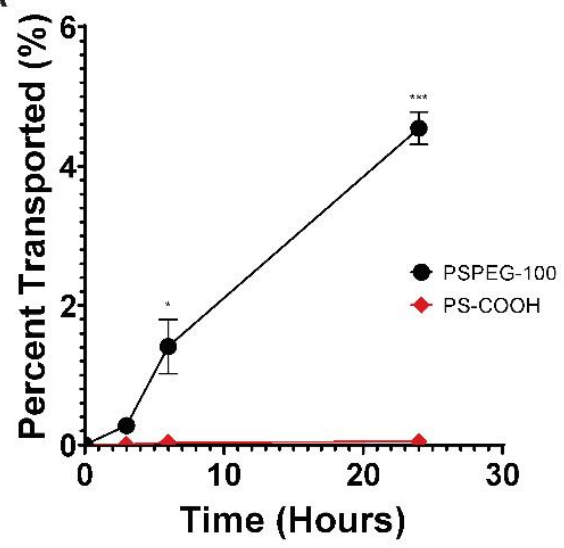

B

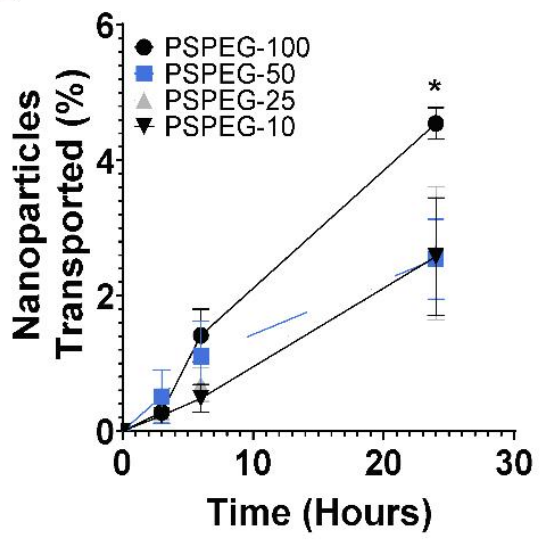

C

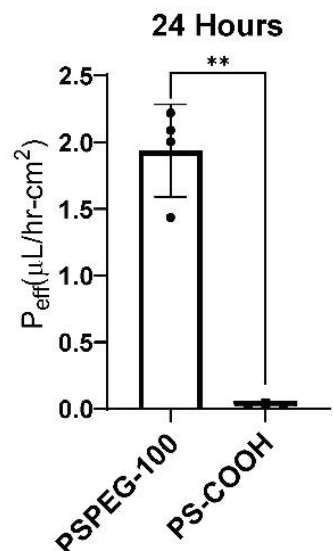

D
Transport at 24 Hours
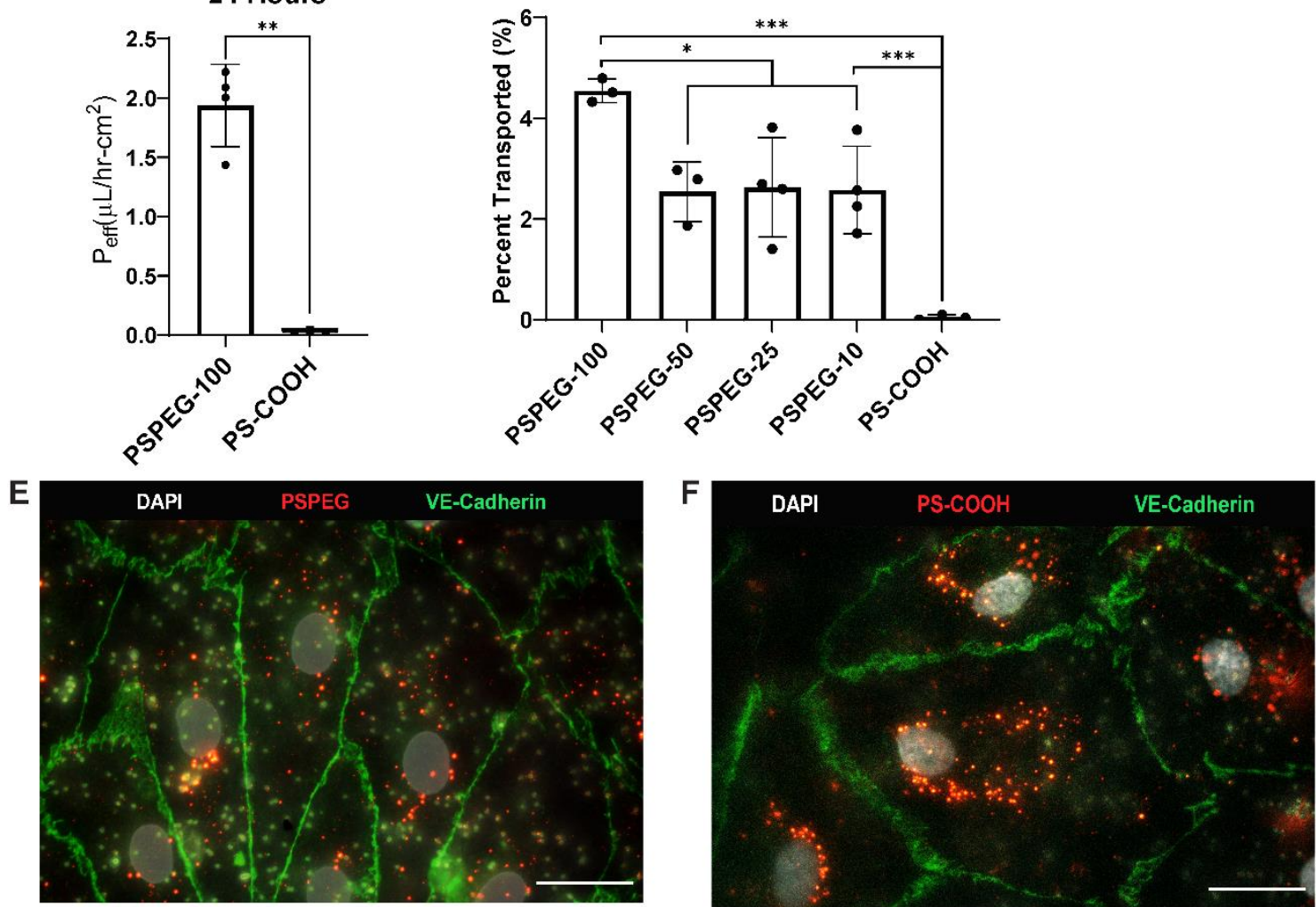

Figure 4: PEGylated nanoparticles transport effectively across primary hLECs. A) Percent of PS and PSPEG-100 nanoparticles transported across hLEC monolayer over time. B) Percent of PSPEG nanoparticles transported after 24 hours. C) Effective permeability $\left(P_{\text {eff }}\right)$ of LEC monolayer to PSPEG and PS nanoparticles. D) Percent of PSPEG transported across LEC monolayer over time. E) Fluorescence images of hLEC, confirmed via VE-cadherin staining (green), treated with PSPEG-100 (red) F) and PS-COOH (red). Scale bars are $20 \mu \mathrm{m}$.

B). All PSPEG were more effectively transported than PS-COOH, and PSPEG-100 transport was

significantly enhanced compared to PSPEG-50, PSPEG-25, and PSPEG-10 after 24 h (Fig 4B). 
After 24 hours, 2.5 - 2.6 $0.9 \%$ PSPEG-50, PSPEG-25, and PSPEG-10 were transported across LECs (Fig 4B). This barrier function of the LEC monolayer can be modeled by measuring the effective permeability $\left(P_{\text {eff }}\right)$ of the system with respect to the nanoparticle system. The measured $P_{\text {eff }}$ of the monolayer to nanoparticles with a dense PEG coating was measured to be $1.9 \pm 0.3$ $\mu \mathrm{L} / \mathrm{hr}^{-\mathrm{cm}^{2}}$ whereas the $\mathrm{P}_{\text {eff }}$ of the monolayer to the PS-COOH nanoparticles was $0.02 \pm 0.02$ $\mu \mathrm{L} / \mathrm{hr}-\mathrm{cm}^{2}$ (Fig 4C). Interestingly, all PSPEG were transported to similar degrees by 6h (Fig 4D). PEGylated and unmodified nanoparticle were internalized by LECs (Fig 4E-F). These results indicate that the addition of PEG enhances the transport of nanoparticles across lymphatics.

\section{Discussion}

In this work, we determined how PEG surface density on nanoparticle affects their transport by lymphatics. We found that addition of PEG to nanoparticle surfaces increased their transport across LECs compared to hydrophobic nanoparticles, and that a dense brush PEG conformation $\left(R_{f} / D>4\right)$ on nanoparticle surface maximized nanoparticle transport by LECs. We also found that increasing the PEG density on the surface of a PS-COOH nanoparticles had measurable differences on nanoparticle properties including size and surface charge, and that none of the changes in material properties resulted in a significant change in nanoparticle uptake by LECs. Finally, we found that while intermediate brush PEG coatings significantly improved nanoparticle transport when compared to un-PEGylated nanoparticles, they were consistently transported less than those nanoparticle with dense brush coatings. Therefore, we here identified that dense brush PEG conformation is required to maximize transport into the lymphatics, which can serve as design criteria for nanoparticle formulations to target lymphatic vessel, and thus the down-stream lymph nodes, for delivery of immune modulatory therapeutics.

PEG conformation on nanoparticle surfaces has been shown to affect nanoparticle uptake by cells. For example, a recent study showed that PEG needed to be in a brush conformation $\left(R_{F} / D\right.$ $\geq 2.8$ ) on the surface of PS- $\mathrm{COOH}$ to evade uptake and clearance by macrophages [20]. Here, authors showed that THP-1 monocytes, preferentially internalized $100 \mathrm{~nm}$ nanoparticles that had 
low PEG grafting density ( $\left.R_{F} / D<2.8\right)$ using low MW PEG (PEG $M W<2$ kDa) [20]. Furthermore, low PEG densities on nanoparticles resulted in rapid clearance of nanoparticles. Another study found that the addition of PEG to the surface of PS-COOH nanoparticle increased their uptake by neutrophils across multiple particles sizes of $2 \mu \mathrm{m}, 500 \mathrm{~nm}$, and $200 \mathrm{~nm}$ [24]. These studies also found no significant difference between the internalization of $200 \mathrm{~nm}$ PS-COOH nanoparticles and PEGylated PS-COOH nanoparticles by THP-1 monocytes, suggesting that PEG density may have a more significant role in modulating uptake by monocytic cells in smaller nanoparticles.

PEG has also been added to nanoparticle surfaces to improve diffusion through endothelial barriers, particularly the blood-brain barrier. A recent study used $100 \mathrm{~nm}$ poly-lactic acid (PLA) PEG nanoparticles with surface PEG ranging from 1 - $10 \mathrm{kDa}$ to probe the effects of PEG MW on transcytosis across brain vascular endothelial monolayers [25]. PEG density on the surface of the nanoparticles was maintained at $17-20 \mathrm{PEG} / 100 \mathrm{~nm}^{2}$. In this study, it was found that the higher molecular weight PEG polymers displayed improved transport across the monolayers, with $60 \%$ translocation efficiency of nanoparticles coated with 5 kDa and $10 \mathrm{kDa}$ MW PEG and only 20\% translocation efficiency of nanoparticles coated with $1 \mathrm{kDa}$ PEG. Interestingly, the maintained PEG density translates to Rf/D > 2 for nanoparticles coated with $5 \mathrm{kDa}$ and $10 \mathrm{kDa}$, while Rf/D < 1 for $1 \mathrm{kDa}$ coated nanoparticles, indicating that PEG on $1 \mathrm{kDa}$ nanoparticles was in mushroom conformation, while it was in brush conformation for $5 \mathrm{kDa}$ and $10 \mathrm{kDa}$ nanoparticles. This suggests that PEG conformation may have a significant role in modulating this transport, consistent with our own findings. Additionally, the authors found that inhibiting micropinocytosis reduced transcytosis by $60 \%$ for $5 \mathrm{kDa}$ PEG coated nanoparticles, suggesting that their transport was mediated at least in part by micropinocytosis. In contrast, transcytosis of $2 \mathrm{kDa}$ PEG coated nanoparticles was reduced only by $25 \%$ after inhibiting micropinocytosis. These findings suggest that uptake and transcytosis pathways may differ with different MW PEG and also PEG conformation on nanoparticles. 
Our investigations on the effect of surface PEG density transport by lymphatic vessels contribute to the growing body of work on engineering nanoparticles to target lymphatic vessel for improved immune modulatory drug delivery to LNs. Recently, $100-150 \mathrm{~nm}$ DPPC liposome surface chemistry was modulated by adding PEG and changing of lipid ratios to yield nanoparticles with PEG coatings ( $\zeta$-potential: $-2.3 \pm 0.5 \mathrm{mV})$, or negative $(-34.3 \pm 9.2 \mathrm{mV})$, neutral $(-1.4 \pm 0.42 \mathrm{mV})$, and positive surface charges $(+26.0 \pm 5.6 \mathrm{mV})$ [26]. These modified nanoparticles were delivered intraperitoneally and the amount of nanoparticles present in lymph fluid and local diaphragmatic lymphatic vessels was measured. Their findings indicated that neutral, negative, and PEGylated nanoparticles effectively entered the lymphatic vessels, followed by transport to the mediastinal LNs and ultimately into systemic circulation via the thoracic duct. Interestingly, positively charged nanoparticles that were retained in the LNs. Notably, PEGylated nanoparticles were retained the least in the LNs, highlighting them as an ideal candidate for the systemic delivery of nanoparticle -based therapy after intraperitoneal administration. Another study used PLGA nanoparticles ranging in size from $50-200 \mathrm{~nm}$ to illustrate that the addition of PEG significantly improved the delivery of nanoparticles to LNs [8]. However, in this study, the surface potential of PEGylated nanoparticles was only $-36.1 \pm 14.6 \mathrm{mV}$, suggesting that the PEG coating was very not dense. This result along with the previous study indicate that hydrophilicity as well as surface charge play a key role in nanoparticle delivery to lymphatics and thus LNs. These findings corroborate our study, where we changed the nanoparticle surface chemistry from hydrophobic to hydrophilic, and from negative to near-neutral charge, all to illustrate that high density surface PEG, in dense brush conformation, maximizes nanoparticle transport by lymphatics.

An alternative route that is being explored for lymphatic targeting is through the gastrointestinal tract, by taking advantage of the chylomicron transport pathways. Lipids are naturally packaged into chylomicron particles by enterocytes, which are transported to systemic circulation via lymphatic vessels. Nanoparticles can be formulated to mimic chylomicrons through the addition of lipids or bile salts on the surface [27-31]. For example, one group generated $100 \mathrm{~nm}$ 
mesoporous silica nanoparticles coated with 1,3-dipalmitoyl-glycerols with a $\zeta$-potential of -8.3 $\mathrm{mV}$, and demonstrated that these nanoparticles entered the lymphatics through the chylomicron pathways after oral administration [27]. Studies using glycocholic acid- (a bile salt) conjugated $100 \mathrm{~nm}$ nanoparticles demonstrated that these nanoparticles improved lymphatic delivery of therapeutics including paclitaxel, insulin, and Exendin-4 compared to uncoated nanoparticles. While these studies primarily focused on characteristics required for uptake by enterocytes, the additional findings from our study can be used to engineer particles that could more effectively enter the lymphatics after chylomicron processing [28-31]. Using the results of our study to generate nanoparticles with dense PEG coating and additionally containing e.g. bile salts or lipids could further improve entry into lymphatics.

In summary, our study demonstrates that the addition of PEG to the surface of hydrophobic nanoparticles improves lymphatic delivery, highlighting this formulation strategy as a promising candidate for designing LN targeted therapeutics. We found that this improved transport into the lymphatics was maximized when the surface PEG on nanoparticles was in a dense brush conformation. Our study is the first to directly correlate PEG density and efficiency of lymphatic transport. Our study examining PEG density and lymphatic transport defines nanoparticle design criteria to enhance drug delivery to the lymphatics and thus to the LNs to enhance immune modulatory therapeutics. 


\section{References}

[1] H. Wiig, M.A. Swartz, Interstitial Fluid and Lymph Formation and Transport: Physiological Regulation and Roles in Inflammation and Cancer, Physiological Reviews 92(3) (2012) 10051060.

[2] S.T. Reddy, A. Rehor, H.G. Schmoekel, J.A. Hubbell, M.A. Swartz, In vivo targeting of dendritic cells in lymph nodes with poly(propylene sulfide) nanoparticles, Journal of Controlled Release 112(1) (2006) 26-34.

[3] V. Manolova, A. Flace, M. Bauer, K. Schwarz, P. Saudan, M.F. Bachmann, Nanoparticles target distinct dendritic cell populations according to their size, European Journal of Immunology 38(5) (2008) 1404-1413.

[4] H. Kobayashi, S. Kawamoto, M. Bernardo, M.W. Brechbiel, M.V. Knopp, P.L. Choyke, Delivery of gadolinium-labeled nanoparticles to the sentinel lymph node: comparison of the sentinel node visualization and estimations of intra-nodal gadolinium concentration by the magnetic resonance imaging, Journal of controlled release : official journal of the Controlled Release Society 111(3) (2006) 343-351.

[5] E.M. Varypataki, A.L. Silva, C. Barnier-Quer, N. Collin, F. Ossendorp, W. Jiskoot, Synthetic long peptide-based vaccine formulations for induction of cell mediated immunity: A comparative study of cationic liposomes and PLGA nanoparticles, Journal of controlled release : official journal of the Controlled Release Society 226 (2016) 98-106.

[6] Q. Zeng, H. Jiang, T. Wang, Z. Zhang, T. Gong, X. Sun, Cationic micelle delivery of Trp2 peptide for efficient lymphatic draining and enhanced cytotoxic T-lymphocyte responses, Journal of Controlled Release 200 (2015) 1-12.

[7] S. De Koker, J. Cui, N. Vanparijs, L. Albertazzi, J. Grooten, F. Caruso, B.G. De Geest, Engineering Polymer Hydrogel Nanoparticles for Lymph Node-Targeted Delivery, Angewandte Chemie International Edition 55(4) (2016) 1334-1339. 
[8] D.A. Rao, M.L. Forrest, A.W.G. Alani, G.S. Kwon, J.R. Robinson, Biodegradable PLGA based nanoparticles for sustained regional lymphatic drug delivery, Journal of pharmaceutical sciences 99(4) (2010) 2018-2031.

[9] J.S. Suk, Q. Xu, N. Kim, J. Hanes, L.M. Ensign, PEGylation as a strategy for improving nanoparticle-based drug and gene delivery, Advanced drug delivery reviews 99(Pt A) (2016) 2851.

[10] S.M. Moghimi, A.C. Hunter, J.C. Murray, Long-circulating and target-specific nanoparticles: theory to practice, Pharmacol Rev 53(2) (2001) 283-318.

[11] F. Alexis, E. Pridgen, L.K. Molnar, O.C. Farokhzad, Factors affecting the clearance and biodistribution of polymeric nanoparticles, Mol Pharm 5(4) (2008) 505-15.

[12] A. Gessner, R. Waicz, A. Lieske, B. Paulke, K. Mäder, R.H. Müller, Nanoparticles with decreasing surface hydrophobicities: influence on plasma protein adsorption, Int J Pharm 196(2) (2000) 245-9.

[13] C.D. Walkey, J.B. Olsen, H. Guo, A. Emili, W.C. Chan, Nanoparticle size and surface chemistry determine serum protein adsorption and macrophage uptake, J Am Chem Soc 134(4) (2012) 2139-47.

[14] H. Otsuka, Y. Nagasaki, K. Kataoka, PEGylated nanoparticles for biological and pharmaceutical applications, Advanced Drug Delivery Reviews 55(3) (2003) 403-419.

[15] J.V. Jokerst, T. Lobovkina, R.N. Zare, S.S. Gambhir, Nanoparticle PEGylation for imaging and therapy, Nanomedicine (London, England) 6(4) (2011) 715-728.

[16] Q. Xu, L.M. Ensign, N.J. Boylan, A. Schön, X. Gong, J.C. Yang, N.W. Lamb, S. Cai, T. Yu, E. Freire, J. Hanes, Impact of Surface Polyethylene Glycol (PEG) Density on Biodegradable Nanoparticle Transport in Mucus ex Vivo and Distribution in Vivo, ACS Nano 9(9) (2015) 921727.

[17] E.A. Nance, G.F. Woodworth, K.A. Sailor, T.-Y. Shih, Q. Xu, G. Swaminathan, D. Xiang, C. Eberhart, J. Hanes, A dense poly(ethylene glycol) coating improves penetration of large 
polymeric nanoparticles within brain tissue, Science translational medicine 4(149) (2012)

149ra119-149ra119.

[18] J.G. Dancy, A.S. Wadajkar, C.S. Schneider, J.R.H. Mauban, O.G. Goloubeva, G.F.

Woodworth, J.A. Winkles, A.J. Kim, Non-specific binding and steric hindrance thresholds for penetration of particulate drug carriers within tumor tissue, Journal of controlled release : official journal of the Controlled Release Society 238 (2016) 139-148.

[19] T.D. Raftery, P. Kerscher, A.E. Hart, S.L. Saville, B. Qi, C.L. Kitchens, O.T. Mefford, T.L. McNealy, Discrete nanoparticles induce loss of Legionella pneumophila biofilms from surfaces, Nanotoxicology 8(5) (2014) 477-84.

[20] Q. Yang, S.W. Jones, C.L. Parker, W.C. Zamboni, J.E. Bear, S.K. Lai, Evading Immune Cell Uptake and Clearance Requires PEG Grafting at Densities Substantially Exceeding the Minimum for Brush Conformation, Molecular Pharmaceutics 11(4) (2014) 1250-1258.

[21] V. Triacca, E. Güç, W.W. Kilarski, M. Pisano, M.A. Swartz, Transcellular Pathways in Lymphatic Endothelial Cells Regulate Changes in Solute Transport by Fluid Stress, Circulation research 120(9) (2017) 1440-1452.

[22] J.L. Perry, K.G. Reuter, M.P. Kai, K.P. Herlihy, S.W. Jones, J.C. Luft, M. Napier, J.E. Bear, J.M. DeSimone, PEGylated PRINT nanoparticles: the impact of PEG density on protein binding, macrophage association, biodistribution, and pharmacokinetics, Nano Lett 12(10) (2012) 530410.

[23] S. Hirosue, E. Vokali, V.R. Raghavan, M. Rincon-Restrepo, A.W. Lund, P. CorthésyHenrioud, F. Capotosti, C. Halin Winter, S. Hugues, M.A. Swartz, Steady-state antigen scavenging, cross-presentation, and CD8+ T cell priming: a new role for lymphatic endothelial cells, J Immunol 192(11) (2014) 5002-11.

[24] W.J. Kelley, C.A. Fromen, G. Lopez-Cazares, O. Eniola-Adefeso, PEGylation of model drug carriers enhances phagocytosis by primary human neutrophils, Acta biomaterialia 79 (2018) 283-293. 
[25] S.F. Tehrani, F. Bernard-Patrzynski, I. Puscas, G. Leclair, P. Hildgen, V.G. Roullin, Length of surface PEG modulates nanocarrier transcytosis across brain vascular endothelial cells, Nanomedicine: Nanotechnology, Biology and Medicine 16 (2019) 185-194.

[26] G. Lee, S. Han, I. Inocencio, E. Cao, J. Hong, A.R.J. Phillips, J.A. Windsor, C.J.H. Porter, N.L. Trevaskis, Lymphatic Uptake of Liposomes after Intraperitoneal Administration Primarily Occurs via the Diaphragmatic Lymphatics and is Dependent on Liposome Surface Properties, Molecular Pharmaceutics 16(12) (2019) 4987-4999.

[27] Y. Mao, S. Feng, S. Li, Q. Zhao, D. Di, Y. Liu, S. Wang, Chylomicron-pretended nano-bio self-assembling vehicle to promote lymphatic transport and GALTs target of oral drugs, Biomaterials 188 (2019) 173-186.

[28] K.S. Kim, K. Suzuki, H. Cho, Y.S. Youn, Y.H. Bae, Oral Nanoparticles Exhibit Specific HighEfficiency Intestinal Uptake and Lymphatic Transport, ACS nano 12(9) (2018) 8893-8900.

[29] K.S. Kim, Y.S. Youn, Y.H. Bae, Immune-triggered cancer treatment by intestinal lymphatic delivery of docetaxel-loaded nanoparticle, Journal of Controlled Release 311-312 (2019) 85-95. [30] J.W. Park, S.J. Kim, D.S. Kwag, S. Kim, J. Park, Y.S. Youn, Y.H. Bae, E.S. Lee, Multifunctional Delivery Systems for Advanced oral Uptake of Peptide/Protein Drugs, Curr Pharm Des 21(22) (2015) 3097-110.

[31] K. Suzuki, K.S. Kim, Y.H. Bae, Long-term oral administration of Exendin-4 to control type 2 diabetes in a rat model, Journal of controlled release : official journal of the Controlled Release Society 294 (2019) 259-267. 